\title{
On constructing Lyapunov functions for multi-agent systems
}

\author{
Hongwei Zhang ${ }^{\mathrm{a} *}$, Zhongkui Li ${ }^{\mathrm{b}}$, Zhihua Qu ${ }^{\mathrm{c}}$, Frank L. Lewis ${ }^{\mathrm{d}, \mathrm{e}}$, Fellow, IFAC \\ ${ }^{a}$ School of Electrical Engineering, Southwest Jiaotong University, Chengdu, 610031, China \\ ${ }^{\mathrm{b}}$ State Key Laboratory for Turbulence and Complex Systems, Department of Mechanics and Engineering Science, College of \\ Engineering, Peking University, Beijing, 100871, China \\ ${ }^{\mathrm{c}}$ Department of Electrical Engineering and Computer Science, University of Central Florida, Orlando, FL, 32816, USA \\ ${ }^{\mathrm{d}}$ UTA Research Institute, University of Texas at Arlington, Fort Worth, TX, 76118, USA
}

${ }^{\text {e } Q i a n ~ R e n ~ C o n s u l t i n g ~ P r o f e s s o r, ~ S t a t e ~ K e y ~ L a b o r a t o r y ~ o f ~ S y n t h e t i c a l ~ A u t o m a t i o n ~ f o r ~ P r o c e s s ~ I n d u s t r i e s, ~ N o r t h e a s t e r n ~ U n i v e r s i t y, ~}$ Shenyang, 110819, China

\begin{abstract}
Lyapunov equations that depend on communication graph topologies provide building blocks of Lypunov functions, which play an important role in controller design and stability analysis of multi-agent systems. However, construction of such a Lyapunov equation in some published works has a flaw or requires unnecessarily strong graph conditions. This paper presents several choices of Lyapunov equations over various graph topologies.
\end{abstract}

Key words: Graph dependent Lyapunov equation, Lyapunov function, multi-agent system, nonnegative graph

\section{Introduction}

Cooperative control of multi-agent systems has attracted a lot of attention in the control community for the past few years, with some representative works being Jadbabaie, Lin, \& Morse (2003); Olfati-Saber, \& Murray (2004); Ren, \& Beard (2005), etc. Stability analysis of multi-agent systems must take into account the connectivity property of the graph, i.e., the way in which the individual systems communicate. The importance in cooperative control stability analysis of constructing a Lyapunov function that depends on communication graph properties is emphasized in Qu (2009); Das, \& Lewis (2010); Zhang, \& Lewis (2012); Zhang, Lewis, \& Qu (2012); Meng, Zhao, \& Lin (2013); Lewis, Zhang,

\footnotetext{
^ This paper was not presented at any IFAC meeting. This work was supported in part by the National Natural Science Foundation of China under grants 61304166, 61433011 and 61473005, the Research Fund for the Doctoral Program of Higher Education under Grant 20130184120013, and by US National Science Foundation under grants ECCS-1308928 and ECCS-1405173.

* Tel.: +86-28-87601026

Email addresses: hwzhangeswjtu.edu.cn (Hongwei Zhang), zhongkli@pku.edu.cn ( Zhongkui Li), qu@eecs.ucf.edu (Zhihua Qu), lewis@uta.edu ( Frank L. Lewis).
}

Hengster-Movric, \& Das (2014), and elsewhere in the literature.

For cooperative tracking problem, a graph dependent Lyapunov equation was proposed in Das, \& Lewis (2010, Lemma 2) when the graph containing all follower nodes is strongly connected. The condition was relaxed in Zhang, Lewis, \& Qu (2012) to allow the graph to have a spanning tree, and further to allow the augmented graph to have a spanning tree (Zhang, \& Lewis, 2012). It was recently noticed that in the Lyapunov equation proposed in Das, \& Lewis (2010, Lemma 2), the construction of the diagonal matrix $P$ can not guarantee a positive definite matrix $Q$ as claimed. A counterexample was given in $\mathrm{Su}, \mathrm{Lin}, \&$ Garcia (2014), which proposed a correct choice of positive definite diagonal matrix $P$ for strongly connected digraphs. However, the graph condition in Su, Lin, \& Garcia (2014) is unnecessarily strong, and can be relaxed. Also, a graph dependent Lyapunov equation proposed in Meng, Zhao, $\&$ Lin (2013) requires the graph to be strongly connected and detailed balanced. This is rather restricted. Considering the importance of graph dependent Lyapunov equations in stability analysis of multi-agent systems, this paper aims to provide a thorough treatment of this issue and lists several Lyapunov equations over different graph topologies, which may be used as building blocks of Lyapunov functions for 
multi-agent systems.

Notations: For notational convenience, 0 denotes either zero scalar, zero vector or zero matrix, according to the context. A vector $x=\left[x_{1}, \ldots, x_{n}\right]^{T}$ is positive, written as $x>0$, if $x_{i}>0$ for all $i$; and nonnegative, written as $x \geq 0$, when all its entries are nonnegative. Matrix $A>0(A \geq 0)$ means that $A$ is positive definite (positive semi-definite); while a positive (nonnegative) matrix is denoted as $A \succ 0(A \succeq 0)$, where all its entries are positive (nonnegative). The spectral radius of matrix $A$ is $\rho(A)$. The empty set is denoted as $\varnothing$. The vector with all entries being ones is $\underline{1}$, and the identity matrix with appropriate dimensions is denoted as $I$.

\section{Preliminaries}

Consider a multi-agent system consisting of $N$ agents (nodes). The communication network among these agents is modeled by a directed graph $\mathcal{G}=\{\mathcal{V}, A\}$, where $\mathcal{V}=\left\{v_{1}, \ldots, v_{N}\right\}$ is the node set and $A=\left[a_{i j}\right] \in \mathbb{R}^{N \times N}$ is the weighted adjacency matrix of the graph, with $a_{i j}$ being the component in the $i$ th row and $j$ th column of $A$. We consider nonnegative graphs, i.e., if there is an edge from node $j$ to node $i$, denoted as $\left(v_{j}, v_{i}\right)$, then $a_{i j}>0$; otherwise, $a_{i j}=0$. Node $j$ is called a neighbor of node $i$, if $a_{i j}>0$. Denote the neighbor set of node $i$ as $\mathcal{N}_{i}=\left\{j \mid a_{i j}>0, j=1,2, \ldots, N\right\}$. We assume the graph is simple, i.e., $a_{i i}=0$. A graph is undirected if $a_{i j}=a_{j i}$ for all $i$ and $j$, and directed otherwise. The graph Laplacian matrix $L=\left[l_{i j}\right] \in \mathbb{R}^{N \times N}$ is defined as

$$
l_{i j}= \begin{cases}-a_{i j}, & j \neq i \\ \sum_{j=1}^{N} a_{i j}, & j=i\end{cases}
$$

A directed path from node $i$ to node $j$ is a sequence of adjacent edges $\left(v_{i}, v_{l}\right),\left(v_{l}, v_{p}\right), \ldots,\left(v_{q}, v_{j}\right)$. A graph has a spanning tree if there is a root node that has a path to every other node. A graph is strongly connected if there is a directed path between every ordered pair of nodes. A graph $\mathcal{G}(A)$ is strongly connected if and only if its adjacency matrix $A$ is irreducible (Berman, \& Plemmons, 1994).

Definition 1 (reducibility/irreducibility) $(Q u, 2009)$ A nonnegative matrix $A=\left[a_{i j}\right] \in \mathbb{R}^{N \times N}$ with $N \geq 2$ is said to be reducible if the set of its indices $\Omega=\{1, \ldots, N\}$ can be divided into two disjoint nonempty sets $\Omega_{p}=\left\{i_{1}, \ldots, i_{p}\right\}$ and $\Omega_{q}=\left\{j_{1}, \ldots, j_{q}\right\}$, satisfying $\Omega_{p} \cup \Omega_{q}=\Omega$ and $\Omega_{p} \cap$ $\Omega_{q}=\varnothing$, such that $a_{i_{\alpha} j_{\beta}}=0$ for all $\alpha=1, \ldots, p$ and $\beta=1, \ldots, q$. Matrix $A$ is irreducible if it is not reducible.

It is widely recognized that Laplacian matrix $L$ plays a fundamental role in stability analysis of multi-agent system. The Laplacian matrix for a nonnegative graph is a singular $\mathrm{M}$ matrix (Qu, 2009). For easy reference, some background of M-matrix and nonnegative matrix are presented here, which will be used in the main results of this paper.
Let $\mathcal{Z}$ be a set of square matrices with nonpositive offdiagonal entries, i.e.,

$$
\mathcal{Z} \triangleq\left\{A=\left[a_{i j}\right] \in \mathbb{R}^{n \times n} \mid a_{i j} \leq 0, \forall i \neq j\right\} .
$$

M-matrix is a subclass of $\mathcal{Z}$, and is defined as below.

Definition 2 (M-matrix) (Berman, \& Plemmons, 1994) A square matrix $A$ is an M-matrix, if it can be expressed in the form

$$
A=s I-C
$$

for some nonnegative matrix $C \succeq 0$ and $s \geq \rho(C)$.

An M-matrix $A=s I-C$ is irreducible (reducible), if the nonnegative matrix $C$ is irreducible (reducible).

Definition 3 (singular/nonsingular M-matrix) (Berman, \& Plemmons, 1994) A square matrix A is a singular M-matrix, if it is an M-matrix with $s \geq \rho(C)$; it is a nonsingular $M$ matrix, if $s>\rho(C)$.

The next result is the well-known Perron-Frobenius theorem, which is a fundamental result on nonnegative matrices.

Lemma $1(Q u, 2009)$ Let $A$ be a nonnegative square matrix.

a) Then $\rho(A) \geq 0$ is an eigenvalue and there exists a nonnegative vectors $x$, such that $A x=\rho(A) x$.

b) If $A \succ 0$, then $\rho(A)>0$ is a simple eigenvalue, and its eigenvector is positive.

c) If $A$ is irreducible, then there exists a positive vector $x$ such that $A x=\rho(A) x$, and $\rho(A)>0$ is a simple eigenvalue of $A$.

Lemma 2 (Berman, \& Plemmons, 1994) A square matrix $A(A \in \mathcal{Z})$ is a nonsingular M-matrix, if and only if one of the following equivalent conditions holds:

a) There is a positive vector $x>0$ such that $A x>0$.

b) There is a positive vector $y>0$ such that $A^{T} y>0$.

c) All the eigenvalues of $A$ have positive real parts.

d) $A$ is nonsingular and $A^{-1}$ is nonnegative.

\section{Constructing Lyapunov functions on graphs}

For cooperative control of multi-agent systems, two extensively studied problems are the leaderless consensus problem (also known as consensus problem, or cooperative regulation problem) and the leader-following consensus problem (or cooperative tracking problem). For the former problem, all agents play an equal role and tend to reach a consensus. For the latter one, there is a leader node, labeled $v_{0}$, whose behavior is not affected by the follower nodes $v_{1}, \ldots, v_{N}$, and all followers are controlled to track the leader node (Lewis, Zhang, Hengster-Movric, \& Das, 2014). In this section, we present a variety of Lyapunov equations on graphs for these two fundamental problems and show briefly how they relate to Lyapunov functions. 


\subsection{Lyapunov equation for leaderless consensus problems}

Proposition 1 ( $Q u, 2009$; Zhang, Lewis, \& Qu, 2012) Suppose that the graph $\mathcal{G}$ is strongly connected. Let $p=\left[p_{1}, \ldots, p_{N}\right]^{T}>0$ be a left eigenvector of the Laplacian matrix L associated with the eigenvalue 0, i.e., $L^{T} p=0$. Define

$$
\begin{aligned}
& P=\operatorname{diag}\left(p_{i}\right), \\
& Q=P L+L^{T} P .
\end{aligned}
$$

Then $P>0$ and $Q \geq 0$.

The existence of such a positive left eigenvector $p$ is guaranteed by Lemma 1 . A normalized $p$ is usually chosen such that $L^{T} p=0$ and $p^{T} \underline{1}=1$. Proposition 1 is proved in Zhang, Lewis, \& Qu (2012) by introducing the concept of generalized Laplacian potential.

The Lyapunov equation (1) can be used to build a Lyapunov function. Take for example the consensus problem of multiagent system with single-integrator dynamics

$$
\dot{x}_{i}=u_{i}, \quad i=1,2, \ldots, N \text {. }
$$

Adopting the well known consensus protocol (Olfati-Saber, \& Murray, 2004)

$$
u_{i}=\sum_{j \in \mathcal{N}_{i}} a_{i j}\left(x_{j}-x_{i}\right)
$$

leads to the closed-loop system

$$
\dot{x}=-L x,
$$

where $x=\left[x_{1}, \ldots, x_{N}\right]^{T}$. It is clear that $V=x^{T} P x$ can serve as a Lyapunov function for system (2), where $P$ solves the Lyapunov equation (1).

Remark 1 When $L$ is an irreducible singular M-matrix in the general sense, not necessarily the Laplacian matrix of a graph, a general Lyapunov equation is provided in $Q u$ (2009, Therorem 4.31) as

$$
\begin{aligned}
& P=\operatorname{diag}\left(y_{i} / x_{i}\right), \\
& Q=P L+L^{T} P,
\end{aligned}
$$

where $x=\left[x_{1}, \ldots, x_{N}\right]^{T}$ and $y=\left[y_{1}, \ldots, y_{N}\right]^{T}$ are the positive right and left eigenvectors of $L$ associated with the eigenvalue 0.

\subsection{Lyapunov equations for leader-following consensus problems}

Denote $\overline{\mathcal{G}}$ as the augmented graph that includes the leader node $v_{0}$, follower nodes $v_{1}, \ldots, v_{N}$, and all associated edges.
The communication between the leader and the followers can be described by a nonnegative matrix $G=\operatorname{diag}\left(g_{i}\right) \succeq$ 0 , where $g_{i}>0$ if and only if there is an edge $\left(v_{0}, v_{i}\right)$. The next graph condition is necessary for leader-following consensus problems (Lewis, Zhang, Hengster-Movric, \& Das, 2014).

Assumption 1 The augmented graph $\overline{\mathcal{G}}$ has a spanning tree, where the leader node $v_{0}$ is the root with no incoming edges from the follower nodes.

Note that this condition does not require the graph $\mathcal{G}$, consisting of all follower nodes, to be strongly connected or have a spanning tree. The following several Lyapunov equations are constructed on graphs satisfying this necessary condition.

Theorem 1 Suppose that Assumption 1 holds. Let

$$
\begin{aligned}
x & =\left[x_{1}, \ldots, x_{N}\right]^{T}=(L+G)^{-1} \underline{1}, \\
y & =\left[y_{1}, \ldots, y_{N}\right]^{T}=(L+G)^{-T} \underline{1}, \\
P & =\operatorname{diag}\left(p_{i}\right)=\operatorname{diag}\left(y_{i} / x_{i}\right), \\
Q & =P(L+G)+(L+G)^{T} P .
\end{aligned}
$$

Then $P>0$ and $Q>0$.

Proof. First, it is trivial to show that $(L+G) \in \mathcal{Z}$, since $L \in \mathcal{Z}$ and $G=\operatorname{diag}\left(g_{i}\right) \succeq 0$. Under Assumption $1, L+G$ is nonsingular (Zhang, \& Lewis, 2012, Lemma 1). Moreover, all its eigenvalues have positive real parts, since $L+G$ is diagonally dominant. Thus $L+G$ is a nonsingular M-matrix, and so is $(L+G)^{T}$. By Lemma 2, we further have that $(L+G)^{-1}$ and $(L+G)^{-T}$ are both nonnegative matrices with no zero rows. This implies that $x>0, y>0$ and $P>0$. Then, obviously, $Q \in \mathcal{Z}$ with nonnegative diagonal entries, since $P=\operatorname{diag}\left(p_{i}\right)>0$. The fact that

$$
\begin{aligned}
Q x & =P(L+G) x+(L+G)^{T} P x \\
& =P \underline{1}+(L+G)^{T} y \\
& =\left[p_{1}, \ldots, p_{N}\right]^{T}+\underline{1} \\
& >0
\end{aligned}
$$

implies, by Lemma 2, that $Q$ is a nonsingular M-matrix and all its eigenvalues have positive real parts. Therefore, $Q$ is positive definite since $Q=Q^{T}$.

For examples of how to construct Lypuanov functions using Lyapunov equation (3), readers are referred to Das, \& Lewis (2010); Zhang, \& Lewis (2012), etc.

Remark 2 In fact, Theorem 1 holds as long as $L+G$ is a nonsingular M-matrix, and $L+G$ does not have to be diagonally dominant, i.e., L may not be a graph Laplacian matrix.

The following result holds immediately, due to the fact that $L+G$ is a nonsingular M-matrix implies that $(L+G)^{T}$ is also a nonsingular M-matrix. 
Corollary 1 Suppose that Assumption 1 holds. Let

$$
\begin{aligned}
x & =\left[x_{1}, \ldots, x_{N}\right]^{T}=(L+G)^{-T} \underline{1}, \\
y & =\left[y_{1}, \ldots, y_{N}\right]^{T}=(L+G)^{-1} \underline{1}, \\
P & =\operatorname{diag}\left(p_{i}\right)=\operatorname{diag}\left(y_{i} / x_{i}\right), \\
Q & =P(L+G)^{T}+(L+G) P .
\end{aligned}
$$

Then $P>0$ and $Q>0$.

Corollary 2 Suppose that Assumption 1 holds, and moreover graph $\mathcal{G}$ is undirected. Let $P=I$. Then

$$
\begin{aligned}
Q & =P(L+G)+(L+G)^{T} P \\
& =P(L+G)^{T}+(L+G) P \\
& >0 .
\end{aligned}
$$

By observing the fact that $L+G$ is diagonally dominant, a special version of Theorem 1 when $x=\underline{1}$ also yields a Lyapunov equation, as follows.

Corollary 3 (Li, Wen, Duan, \& Ren, 2015) Suppose that Assumption 1 holds. Let

$$
\begin{aligned}
p & =\left[p_{1}, \ldots, p_{N}\right]^{T}=(L+G)^{-T} \underline{1}, \\
P & =\operatorname{diag}\left(p_{i}\right), \\
Q & =P(L+G)+(L+G)^{T} P .
\end{aligned}
$$

Then $P>0$ and $Q>0$.

Proof. As shown in Theorem $1, L+G$ is a nonsingular Mmatrix and $P$ is a positive diagonal matrix. Since $L+G$ is diagonally dominant, $(L+G) \underline{1} \geq 0$, and thus

$$
P(L+G) \underline{1} \geq 0 .
$$

Also we have

$$
(L+G)^{T} P \underline{1}=(L+G)^{T} p=\underline{1}>0 .
$$

Then we have $Q \underline{1}>0$. By Lemma $2, Q$ is a nonsingular M-matrix and all its eigenvalues have positive real parts. Therefore, $Q$ is positive definite since $Q=Q^{T}$.

Remark 3 The same construction was also proposed in $\mathrm{Su}$, Lin, \& Garcia (2014) under the assumption that the graph $\mathcal{G}$ is strongly connected and at least one follower node obtains information of the leader, i.e., $g_{i}>0$ for at least one $i$. Compared with Assumption 1, this graph condition is unnecessarily strong. However, under this restricted graph condition, we can construct alternative Lyapunov equations as below, which get rid of the matrix inverse operation existing in Theorem 1 and its corollaries.

Theorem 2 Suppose that Assumption 1 holds and more specifically graph $\mathcal{G}$ is strongly connected. Denote $\lambda=\max _{i}\left(l_{i i}+g_{i}\right)$ and $B=\lambda I-(L+G)$. Let $x>0$ and $y>0$ be the first right and left eigenvectors of $L+G$ with respect to the eigenvalue $\lambda-\rho(B)$, i.e.,

$$
\begin{aligned}
& (L+G) x=(\lambda-\rho(B)) x \\
& (L+G)^{T} y=(\lambda-\rho(B)) y .
\end{aligned}
$$

Define

$$
\begin{aligned}
& P=\operatorname{diag}\left(y_{i} / x_{i}\right), \\
& Q=P(L+G)+(L+G)^{T} P .
\end{aligned}
$$

Then $P>0$ and $Q>0$.

Proof. Since $\mathcal{G}$ is strongly connected, $B$ is nonnegative and irreducible. By Lemma $1, \rho(B)$ is a simple eigenvalue of $B$ and there is a positive vector $x>0$ such that $B x=\rho(B) x$. Similarly, there exists $y>0$ such that $B^{T} y=\rho\left(B^{T}\right) y=$ $\rho(B) y$. Clearly, $\lambda-\rho(B)>0$ is the first eigenvalue of $L+G$, since $L+G$ is a nonsingular M-matrix. Following similar development as in Theorem 1 , we can show that $Q>0$.

Corollary 4 Suppose that Assumption 1 holds and the graph $\mathcal{G}$ is strongly connected. Denote $\lambda=\max _{i}\left(l_{i i}+g_{i}\right)$ and $B=\lambda I-(L+G)$. Let $x>0$ and $y>0$ be the first right and left eigenvectors of $L+G$, i.e.,

$$
\begin{aligned}
& (L+G) x=(\lambda-\rho(B)) x, \\
& (L+G)^{T} y=(\lambda-\rho(B)) y .
\end{aligned}
$$

Define

$$
\begin{aligned}
& P=\operatorname{diag}\left(x_{i} / y_{i}\right), \\
& Q=P(L+G)^{T}+(L+G) P,
\end{aligned}
$$

Then $P>0$ and $Q>0$.

Under more restricted graph condition, Meng, Zhao, \& Lin (2013) proposed the following Lyapunov equation.

Proposition 2 Suppose that Assumption 1 holds. We further assume that the graph $\mathcal{G}$ is not only strongly connected, but also detailed balanced, i.e.,

$$
p_{i} a_{i j}=p_{j} a_{j i}, \quad \forall i, j
$$

where $p_{i}>0$ for all $i$. A Lyapunov equation can be constructed as

$$
Q=P(L+G)+(L+G)^{T} P
$$

where $P=\operatorname{diag}\left(p_{i}\right)>0$. Then $Q>0$. 


\section{Conclusion}

The construction of graph dependent Lyapunov equations reported in some published works has a flaw due to the improper choice of the diagonal matrix $P$, or requires unnecessarily strong graph conditions (e.g., Das, \& Lewis, 2010; Zhang, \& Lewis, 2012; Meng, Zhao, \& Lin, 2013; Su, Lin, \& Garcia, 2014). This paper provides a thorough treatment of constructing graph dependent Lyapunov equations, which provides a building block of Lyapunov functions, and thus should facilitate the stability analysis for cooperative control of multi-agent systems.

\section{References}

Berman, A. \& Plemmons, R. (1994). Nonnegative Matrices in the Mathematical Sciences. Society for Industrial and Applied Mathematics, Philadelphia.

Das, A. \& Lewis, F.L. (2010). Distributed adaptive control for synchronization of unknown nonlinear networked systems. Automatica, 46(12), 2014-2021.

Jadbabaie, A., Lin, J., \& Morse, A.S. (2003). Coordination of groups of mobile autonomous agents using nearest neighbor rules. IEEE Transactions on Automatic Control, 48(6), 988-1001.

Lewis, F.L., Zhang, H., Hengster-Movric, K., \& Das, A. (2014). Cooperative Control of Multi-Agent Systems: Optimal and Adaptive Design Approaches. Springer-Verlag, London.

Li, Z., Wen, G., Duan, Z., \& Ren, W. (2015). Designing fully distributed consensus protocols for linear multi-agent systems with directed graphs. IEEE Transactions on Automatic Control, 60(4), 1152-1157.

Meng, Z., Zhao, Z., \& Lin, Z. (2013). On global leaderfollowing consensus of identical linear dynamic systems subject to actuator saturation. Systems \& Control Letters, 62(2), 132-142.

Olfati-Saber, R., \& Murray, R. (2004). Consensus problems in networks of agents with switching topology and timedelays. IEEE Transactions on Automatic Control, 49(9), 1520-1533.

Qu, Z. (2009). Cooperative Control of Dynamical Systems: Applications to Autonomous Vehicles. Springer-Verlag, London.

Ren, W., \& Beard, R. (2005). Consensus seeking in multiagent systems under dynamically changing interaction topologies. IEEE Transactions on Automatic Control, 50(5), 655-661.

Su, S., Lin, Z., \& Garcia, A. (2014). Distributed synchronization control of multi-agent systems with unknown nonlinearities: the case of fixed directed communication topology. in Proceedings of the American Control Conference (pp. 5361-5366).

Zhang, H., \& Lewis, F.L. (2012). Adaptive cooperative tracking control of higher-order nonlinear systems with unknown dynamics. Automatica, 48(7), 1432-1439.

Zhang, H., Lewis, F.L., \& Qu, Z. (2012). Lyapunov, adaptive, and optimal design techniques for cooperative systems on directed communication graphs. IEEE Transactions on Industrial Electronics, 59(7), 3026-3041. 